\title{
Scaling of the Fano Effect of the In-Plane Fe-As Phonon and the Superconducting Critical Temperature in $\mathrm{Ba}_{1-x} \mathrm{~K}_{x} \mathrm{Fe}_{2} \mathrm{As}_{2}$
}

\author{
B. Xu,${ }^{1, *}$ E. Cappelluti, ${ }^{2}$ L. Benfatto, ${ }^{3}$ B. P. P. Mallett, ${ }^{1,4}$ P. Marsik, ${ }^{1}$ E. Sheveleva, ${ }^{1}$ F. Lyzwa, ${ }^{1}$ \\ Th. Wolf, ${ }^{5}$ R. Yang, ${ }^{6}$ X. G. Qiu, ${ }^{6}$ Y. M. Dai, ${ }^{7}$ H. H. Wen, ${ }^{7}$ R. P. S. M. Lobo, ${ }^{8,9}$ and C. Bernhard ${ }^{1, \dagger}$ \\ ${ }^{1}$ University of Fribourg, Department of Physics and Fribourg Center for Nanomaterials, \\ Chemin du Musée 3, CH-1700 Fribourg, Switzerland \\ ${ }^{2}$ Istituto di Struttura della Materia, CNR, 34149 Trieste, Italy \\ ${ }^{3}$ ISC-CNR and Department of Physics, Sapienza University of Rome, P. le A. Moro 5, 00185 Rome, Italy \\ ${ }^{4}$ The Photon Factory, Department of Physics, University of Auckland, 38 Princes Street, Auckland 1010, New Zealand \\ ${ }^{5}$ Institute of Solid State Physics, Karlsruhe Institute of Technology, Postfach 3640, Karlsruhe 76021, Germany \\ ${ }^{6}$ Beijing National Laboratory for Condensed Matter Physics, Institute of Physics, Chinese Academy of Sciences, Beijing 100190, China \\ ${ }^{7}$ National Laboratory of Solid State Microstructures and Department of Physics, Nanjing University, Nanjing 210093, China \\ ${ }^{8}$ LPEM, ESPCI Paris, PSL University, CNRS, F-75005 Paris, France \\ ${ }^{9}$ Sorbonne Université, CNRS, LPEM, F-75005 Paris, France
}

(Received 18 October 2018; published 29 May 2019)

\begin{abstract}
By means of infrared spectroscopy, we determine the temperature-doping phase diagram of the Fano effect for the in-plane Fe-As stretching mode in $\mathrm{Ba}_{1-x} \mathrm{~K}_{x} \mathrm{Fe}_{2} \mathrm{As}_{2}$. The Fano parameter $1 / q^{2}$, which is a measure of the phonon coupling to the electronic particle-hole continuum, shows a remarkable sensitivity to the magnetic and structural orderings at low temperatures. Most strikingly, at elevated temperatures in the paramagnetic tetragonal state we observe a linear correlation between $1 / q^{2}$ and the superconducting critical temperature $T_{c}$. Based on theoretical calculations and symmetry considerations, we identify the relevant interband transitions that are coupled to the Fe-As mode. In particular, we show that a sizable $x y$ orbital component at the Fermi level is fundamental for the Fano effect and, thus, possibly also for the superconducting pairing.
\end{abstract}

DOI: 10.1103/PhysRevLett.122.217002

The identification of the superconducting (SC) pairing mechanism of the iron arsenides is complicated by their multiband and multigap structure and by the entanglement of the magnetic, orbital, and structural degrees of freedom [1-7]. A prominent example is the stripelike antiferromagnetic (AFM) order in undoped and weakly doped $\mathrm{Ba}_{1-x} \mathrm{~K}_{x} \mathrm{Fe}_{2} \mathrm{As}_{2}$ (BKFA), described by a single $q$ vector along $(0, \pi)$, that is accompanied by an orthorhombic distortion of the high-temperature tetragonal structure with strong nematic fluctuations [7]. This orthorhombic AFM (o-AFM) order persists well into the SC regime, where it coexists with superconductivity at $0.15 \leq x \leq 0.3$. The strongest $\mathrm{SC}$ response, in terms of the $T_{c}$ value [8,9], condensate density [10], and condensation energy [11], occurs around optimum doping $(x=0.3-0.35)$ just as the $\mathrm{o}-\mathrm{AFM}$ order vanishes. A further anomaly in $T_{c}$ is observed at doping $x=0.24-0.26[9,10,12]$, where a different AFM order with tetragonal symmetry (t-AFM) occurs and competes with the o-AFM state $[8,13-18]$.

The strong electronic correlations and, in particular, magnetic fluctuations have also pronounced effects on the band structure in the vicinity of the Fermi surface (FS). In BKFA, the FS is composed of several hole pockets at the center of the Brillouin zone (BZ) ( $\Gamma$ point) and electron pockets at the zone boundary ( $M$ point) that have mainly Fe $x z, y z$, and $x y$ character. These bands are about 2 times narrower than those predicted by density-functional theory calculations $[19,20]$ and, in addition, are pushed toward the FS [21]. The latter effect arises from the strong particle-hole asymmetry and a pronounced interband scattering between the $M$ and $\Gamma$ points that most likely involves AFM fluctuations [22-24]. Especially near the $M$ point, this yields very flat bands in the vicinity of the FS with a nearly singular behavior that is very pronounced in the angle-resolved photoemission spectroscopy (ARPES) spectra of optimal doped Sm-1111 and BKFA $[21,25]$.

Although the bare electron-phonon coupling is commonly believed to be very weak [26], it can be strongly increased by the spin-phonon interaction [27-29]. This could explain why a strong enhancement of the Fano effect of the in-plane $E_{u} \mathrm{Fe}$-As mode was observed in the o-AFM state of undoped $\mathrm{BaFe}_{2} \mathrm{As}_{2}$ [30-32]. On the other hand, a sizable Fano effect was also reported for optimal doped BKFA without AFM order [33,34]. To the best of our knowledge, a systematic study of the evolution of this Fano effect as a function of the doping and temperature and an assignment of the underlying electronic and magnetic excitations are still lacking. 

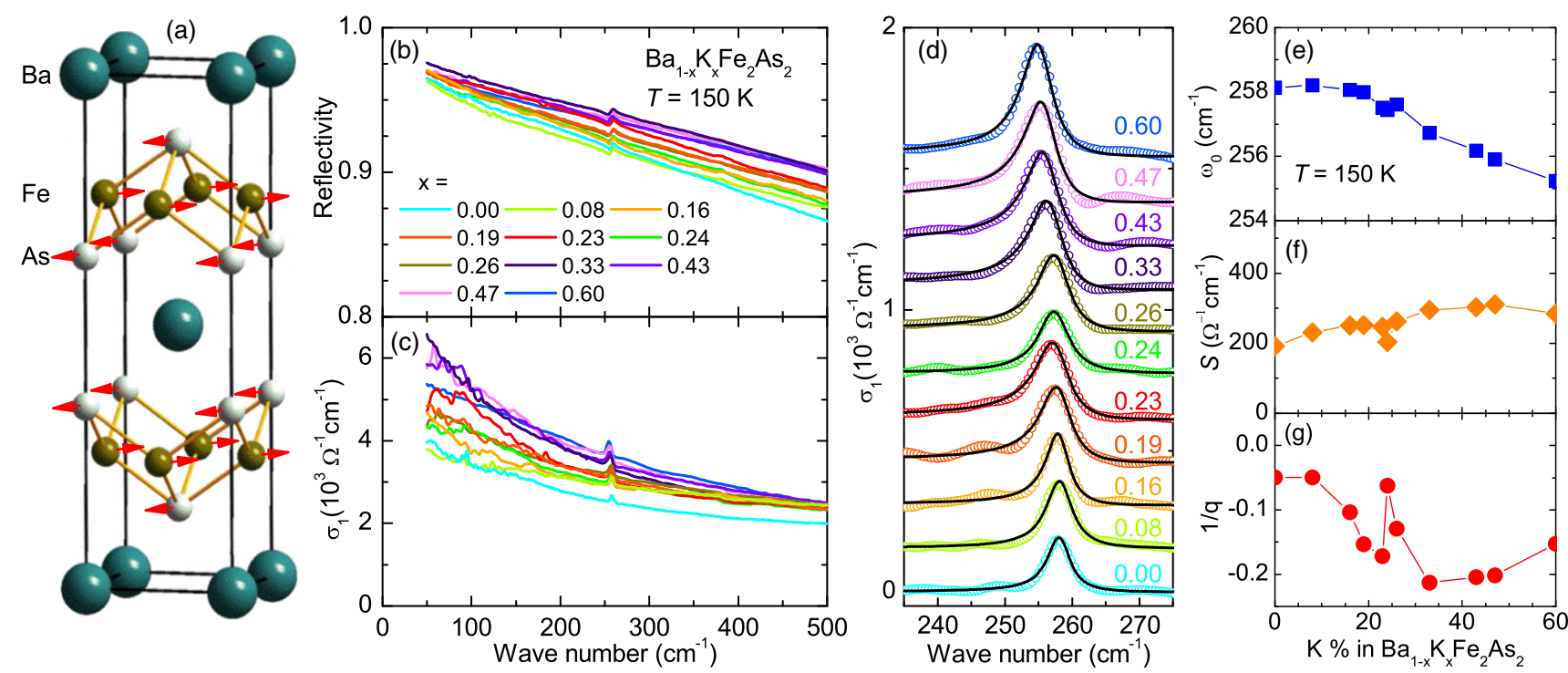

FIG. 1. (a) Structure of $\mathrm{BaFe}_{2} \mathrm{As}_{2}$ and eigenvectors of the Fe-As stretching mode. Doping dependence of (b) the reflectivity and (c) the corresponding optical conductivity at $T=150 \mathrm{~K}$ in $\mathrm{Ba}_{1-x} \mathrm{~K}_{x} \mathrm{Fe}_{2} \mathrm{As}_{2}$. (d) Enlarged view of the phonon (with offsets). The black solid lines through the data show the corresponding Fano fits. (e)-(g) Doping dependence of the phonon frequency $\omega_{0}$, the oscillator strength $S$, and the Fano parameter $1 / q$ of the $E_{u}$ phonon in $\mathrm{Ba}_{1-x} \mathrm{~K}_{x} \mathrm{Fe}_{2} \mathrm{As}_{2}$ at $150 \mathrm{~K}$.

Here we close this knowledge gap by showing the detailed temperature and doping dependence of the Fano effect of the $E_{u}$ mode in BKFA. A sketch of the structure and the eigenvectors of the phonon is shown in Fig. 1(a). At low temperatures, the strength of the Fano coupling, expressed in terms of the asymmetry parameter $1 / q^{2}$, appears to be very sensitive to the magnetic and structural transitions at $x<0.3$. Most remarkably, at temperatures well above these magnetic and structural transitions (in the paramagnetic tetragonal state), we find that $1 / q^{2}$ exhibits a linear scaling with the SC critical temperature $T_{c}$. This striking observation is suggestive of an intimate relationship between the electronic excitations coupled to the $E_{u}$ phonon mode and the SC pairing mechanism.

The BKFA single crystals with $0 \leq x \leq 0.6$ were grown with a flux method [35]. Their $\mathrm{K}$ content was determined with $\mathrm{x}$-ray profile refinement and electron dispersive $\mathrm{x}$-ray spectroscopy to an accuracy of $\Delta x \approx 0.02[10,12]$. The AFM and SC transition temperatures $T_{N}$ and $T_{c}$ were derived from the anomalies in the dc resistivity curves. The $a b$-plane reflectivity $R(\omega)$ was measured at near-normal incidence with a Bruker VERTEX $70 \mathrm{~V}$ spectrometer. An in situ gold overfilling technique [36] was used to obtain the absolute reflectivity of the samples. The room temperature spectrum in the near infrared to ultraviolet (4000-50000 $\mathrm{cm}^{-1}$ ) was measured with a commercial ellipsometer (Woollam VASE). The optical conductivity was obtained from a Kramers-Kronig analysis of $R(\omega)$ [37]. For the extrapolation at low frequency, we used the function $R=1-A \sqrt{\omega}$ (Hagen-Rubens) in the normal state and $R=1-A \omega^{4}$ in the SC state. On the high-frequency side, we used the room temperature ellipsometry data and extended them by assuming a constant reflectivity up to $12.5 \mathrm{eV}$ that is followed by a free-electron $\left(\omega^{-4}\right)$ response.

Figures 1(b) and 1(c) show the doping-dependent reflectivity and conductivity spectra, respectively, of BKFA in the paramagnetic state, for a wide doping range from undoped to strongly overdoped. The curves of $R(\omega)$ are fairly similar and increase toward low frequency, where they approach unity at zero frequency, indicating a typical metallic behavior. The corresponding spectra of $\sigma_{1}(\omega)$ at $150 \mathrm{~K}$ in Fig. 1(c) are dominated by the strong electronic response that is composed of a Drude peak at the origin, due to the itinerant carriers, and a pronounced tail toward high frequency, that arises from inelastic scattering of the free carriers and/or low-lying interband transitions [38-41]. The full temperature and doping dependence of the $\sigma_{1}(\omega)$ spectra, showing how the electronic response gets modified in the AFM and/or SC states, can be found in Supplemental Material [42]. These spectra agree well with previous reports [10,43-47] and reveal a spin-density-wave gap below the Neél temperature $T_{N}$ and a superconducting gap below $T_{c}$.

In addition to these broad electronic features, the infrared-active Fe-As stretching mode (with $E_{u}$ symmetry) [30] is clearly visible in all $R(\omega)$ and $\sigma_{1}(\omega)$ spectra as a weak but sharp mode around $255 \mathrm{~cm}^{-1}$. The remarkable doping dependence of this phonon mode is detailed in Fig. 1(d), which shows a magnified view of the Fe-As phonon mode with the electronic background subtracted. The subtraction of the electronic background and its influence on the phonon line shape is discussed in Supplemental Material [42]. For a quantitative analysis, we fitted [solid lines in 

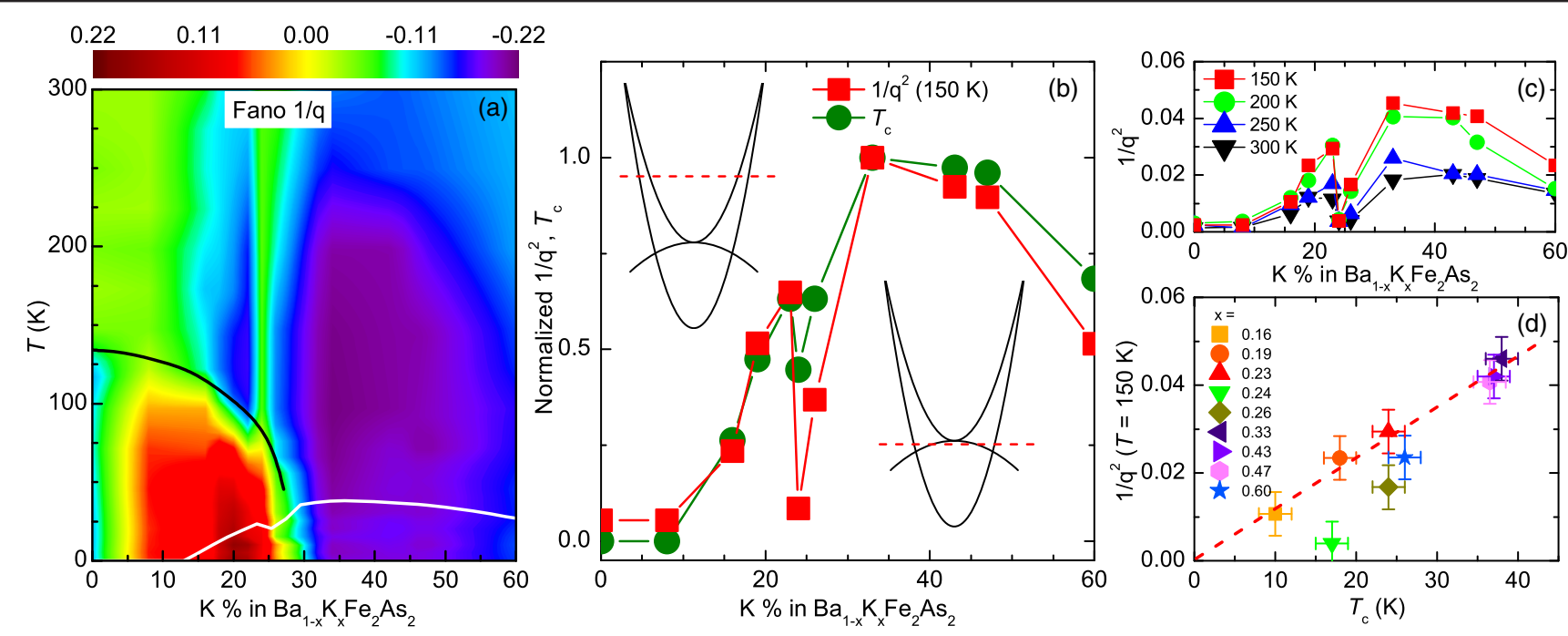

FIG. 2. (a) Color map of the Fano parameter $1 / q$ in the $T$ vs $x$ plane for $\mathrm{Ba}_{1-x} \mathrm{~K}_{x} \mathrm{Fe}_{2} \mathrm{As}_{2}$. The solid black and white lines mark the AFM and SC transition temperatures, respectively. (b) Doping dependence of the Fano parameter $1 / q^{2}$ at $T=150 \mathrm{~K}$ and the SC transition temperature $T_{c}$ (both normalized to their maximum value). Also shown is a sketch of the doping dependence of the band structure around the $M$ points, according to ARPES data [21,25]. The dashed red line denotes the Fermi level, that around optimal doping crosses the flat sections of the bands, where the density of states is higher. (c) Doping dependence of the Fano parameter $1 / q^{2}$ at $T=150,200,250$, and $300 \mathrm{~K}$. (d) Scaling of the Fano parameter 1/q vs the SC transition temperature $T_{c}$ in $\mathrm{Ba}_{1-x} \mathrm{~K}_{x} \mathrm{Fe}_{2} \mathrm{As}_{2}$.

Fig. 1(d)] the optical properties of the $E_{u}$ mode with a Fano function $[48-50]$ :

$$
\sigma_{1}(\omega)=S\left(\frac{q^{2}+2 q z-1}{q^{2}\left(1+z^{2}\right)}\right),
$$

where $z=\left(\omega-\omega_{0}\right) / \Gamma$ and where $\omega_{0}, \Gamma$, and $S$ are the frequency, linewidth, and strength of the phonon mode, respectively. An asymmetric profile of the phonon line shape is ruled here by the Fano parameter $1 / q^{2}$, which reflects the strength of the coupling between the phonon and the underlying optical electronic excitations. When the electronic excitations are lacking or they are not coupled with the phonon resonance, one has $1 / q^{2}=0$ and a symmetric Lorentzian line shape is recovered.

The obtained phonon parameters $\omega_{0}, S$, and $1 / q$ at $150 \mathrm{~K}$ are shown in Figs. 1(e)-1(g). With K doping, $\omega_{0}$ moves to a lower frequency (redshift) and $S$ increases. More strikingly, the Fano parameter $1 / q$ shows a significant doping dependence. In the following, we focus on the evolution of this parameter. The temperature dependence of the phonon mode and its parameters for different samples in BKFA are further detailed in Supplemental Material [42]. Here, the full doping and temperature dependence of $1 / q$ is summarized as a color map in Fig. 2(a). Also shown is the evolution of $T_{N}$ (solid black line), which is accompanied by abrupt changes of $1 / q$, and of $T_{c}$ (solid white line), which hardly affects the $1 / q$ value. In the paramagnetic state, $1 / q$ is negative for all samples [as also shown in Fig. 1(g)], which suggests that the phonon mode is coupled to electronic interband transitions mainly centered at a higher energy than the phonon. The abrupt sign reversal of $1 / q$ below $T_{N}$ has a natural explanation in the band folding induced by the AFM state, shifting the weight of the coupled optical transitions at energies $\omega<\omega_{0}$. Such optical transitions at a low energy activated by band folding in the AFM state are clearly visible at $\omega \approx 200 \mathrm{~cm}^{-1}$ in Fig. S1(c) $(x=0.19)$ in Supplemental Material [42] and partially at $\omega \approx 120 \mathrm{~cm}^{-1}$ for $x=0.08$ [Fig. S1(b)]. A further detailed analysis is provided in Supplemental Material [42]. At $x=0$, it was furthermore shown that this backfolding leads to Dirac cones close to the FS which rapidly move above the FS upon hole doping [32,51]. Accordingly, at $x=0$ the negative $1 / q$ below $T_{N}$ is caused by a Van Hove singularity that is related to the Dirac cones and is seen as a cusp feature in $\sigma_{1}(\omega)$ around $350 \mathrm{~cm}^{-1}$ [32].

In the following, we focus on the doping dependence of $1 / q^{2}$ without the influence of the AFM and structural transitions as derived from the constant-temperature cut in the paramagnetic tetragonal phase. The Fano parameter $1 / q^{2}$ at $T=150 \mathrm{~K}$ in Fig. 2(b) reveals a characteristic domelike profile that closely resembles the one of $T_{c}$. Notably, this characteristic profile persists up to $300 \mathrm{~K}$ [see Fig. 2(c)], suggesting that it is not caused by fluctuations that occur close to one of the low-temperature phase transitions. An anomaly at $x=0.24-0.26$ is also observed in $1 / q^{2}$ in correspondence with the one of $T_{c}$ and persists up to room temperature. Such a sudden anomaly at $x=0.24-0.26$, i.e., close to quarter filling, is reminiscent of the stripe-ordering instability in the cuprates at $x \approx 1 / 8$ doping [52,53]. It implies that at this particular doping an additional interaction or instability (that remains to be 
identified) affects the normal state properties as much as the magnetic and superconducting ones.

A striking almost linear correlation in the doping range $x=0-0.6$ between the Fano parameter of the $E_{u}$ mode in the paramagnetic state and the $\mathrm{SC}$ transition temperature is further highlighted in Fig. 2(d), where we plot $1 / q^{2}$ vs $T_{c}$. Once more, a clear deviation from a linear behavior occurs only for the $x=0.24$ and 0.26 samples.

To shed more light on the physical processes responsible for the Fano effect of the $E_{u}$ Fe-As mode in these materials, we analyzed the optical data within the charged-phonon scheme that was originally developed for carbon-based materials $[49,50,54,55]$. In this context, the Fano asymmetry parameter $1 / q$ is essentially ruled by the imaginary part of a complex function $\chi(\omega)$ that can be identified as a dynamical response function between the current operator and the electron-phonon operator relative to the $E_{u}$ phonon. In particular, $1 / q$ scales as the imaginary part at the phonon frequency $\omega_{0}$, i.e., $1 / q \propto \operatorname{Im} \chi\left(\omega_{0}\right)$, which is different from zero whenever the $E_{u}$ phonon is coupled to an electronic interband transition at $\omega_{0}$. To identify the relevant processes responsible for the Fano effect, we analyze the paramagnetic tetragonal state without any structural distortion. Using the Slater-Koster approach [56,57], we consider a minimal tight-binding model containing the three orbitals $(x z, y z, x y)$ relevant for the description of the low-energy band structure in the doping range under consideration. We compute the electron-phonon operator for the $E_{u}$ mode at linear order in the lattice displacement, and we analyze the properties of the corresponding charged-phonon response function $\chi(\omega)$. As detailed in Supplemental Material [42], we find that, when only the $x z / y z$ orbital subsector is considered, the charged-phonon response function $\chi(\omega)$ is vanishing at the leading order, implying a corresponding vanishing Fano effect. This property is a consequence of the underlying symmetries of the $x z / y z$ subsystem. On the other hand, a finite Fano asymmetry is possible when the $x y$ orbital component is taken into account, allowing for a finite contribution to $\chi(\omega)$ of interband particle-hole transitions between the $x y$ and $x z / y z$ components of the bands. Even though a quantitative estimate of $\chi\left(\omega_{0}\right)$ at the $E_{u}$ phonon frequency is beyond the scope of the present Letter, we can nonetheless conclude that the contribution of the $x y$ orbital to the low-energy optical transitions plays a dominant role in the experimentally observed domelike doping dependence of the Fano asymmetry.

These conclusions hold true as long as the abovementioned symmetries are preserved. In this respect, any breaking of the $x z / y z$ equivalence due, e.g., to electronic or structural nematic phases can prompt additional low-energy transitions, leading to an otherwise forbidden Fano-type coupling with the $E_{u}$ mode. We believe that this is the case, in particular, for the AFM ordered states. Note, on the other hand, that the SC phase does not modify the symmetries ruling the charged-phonon response, explaining the observed lack of a significant change of the $E_{u}$ mode in the SC state.

The primary role of the low-energy transitions between the $d_{x y}$ and the $d_{x z / y z}$ bands for understanding the phase diagram of the Fano effect of the $E_{u}$ mode and the remarkable correlation between the Fano parameter $1 / q^{2}$ and the SC critical temperature $T_{c}$ reported in Fig. 2(d) call for a closer look into the band structure and its doping evolution. Such optical transitions at a low energy in the vicinity of the $E_{u}$ phonon occur only close to the $M$ points, where recent ARPES data [21,25] showed that as doping increases the Fermi level is pushed towards the bottom of the electronlike bands [see Fig. 2(b)], so that the Fermi surface at the $M$ points evolves from an elliptical shape to electronlike propellers. In this regime, the bands close to the $M$ points are remarkably flat, leading to a substantial increase of the density of states. This can, in turn, affect the joint density of states of the optical interband transitions near the $M$ points, that govern the Fano effect, but also the strength of the AFM spin-fluctuation exchange between the $M$ and $\Gamma$ pockets, pointing towards their predominance as a source of pairing glue. It is also worth mentioning that several recent theoretical studies [24,58-63] pointed out that a correct description of the spin-fluctuation exchange between hole and electron pockets cannot leave aside the orbital composition of the bands. Indeed, since the microscopic interactions are primarily intraorbital in character, the spin-mediated interpocket exchange is possible only when the same orbital component appears in both pockets. As a consequence, the flattening of the $x y$ bands at the $M$ points can also explain a sizable spin-fluctuation exchange between the $M$ pockets [59], which provides a secondary $d$ wave pairing channel with respect to the dominant $s_{ \pm}$one, boosting $T_{c}$. Interestingly, this mechanism has been recently invoked to explain the appearance below $T_{c}$ of excitoniclike Bardasis-Schrieffer resonances in the Raman spectra $[64,65]$. Finally, whereas it seems unlikely that the electron-phonon coupling is the primary mechanism of the SC pairing, it remains to be seen whether the $E_{u}$ phonon is just an "accidental witness" of the SC pairing interaction (via its sensitivity to the $x y$ orbital content at the Fermi level) or whether it even plays a cooperative role and enhances $T_{c}$.

In summary, we performed a systematic study of the Fano effect of the in-plane Fe-As stretching mode in $\mathrm{Ba}_{1-x} \mathrm{~K}_{x} \mathrm{Fe}_{2} \mathrm{As}_{2}$. First, we showed that the Fano effect of this phonon mode is strongly enhanced by the magnetic and structural transitions into the orthorhombic AFM state. Second, and most importantly, we observed a striking linear relationship between the Fano parameter $1 / q^{2}$, as measured at temperatures well above the magnetic and structural transitions, and the SC critical temperature $T_{c}$. Theoretical calculations based on symmetry considerations show that the $x y$ orbital component of the low-energy bands near the $M$ point of the BZ plays a central role for the Fano effect of 
the $E_{u}$ phonon mode. This calls for a detailed investigation of the role played by the same orbital degrees of freedom on the orbital-selective pairing mechanism based on spinfluctuation exchange and their possibly cooperative interplay with electron-phonon coupling.

Work at the University of Fribourg was supported by the Schweizerische Nationalfonds (SNF) by Grant No. 200020172611. L. B. acknowledges financial support by the Italian MAECI under the Italian-India collaborative Project No. SUPERTOP-PGR04879. Work at IOP was supported by MOST of China (Grant No. 2017YFA0302903) and NSFC of China (Grant No. 11774400). Work at Nanjing University was supported by the project of MOST of China (Grant No. 2016YFA0300401) and NSFC of China (Grants No. 11534005 and No. 11874206). B. P. P. M. acknowledges support from the Rutherford Foundation.

*bing.xu@unifr.ch

†christian.bernhard@unifr.ch

[1] J. Paglione and R. L. Greene, Nat. Phys. 6, 645 (2010).

[2] G. R. Stewart, Rev. Mod. Phys. 83, 1589 (2011).

[3] I. I. Mazin, Nature (London) 464, 183 (2010).

[4] F. Wang and D.-H. Lee, Science 332, 200 (2011).

[5] A. Chubukov, Annu. Rev. Condens. Matter Phys. 3, 57 (2012).

[6] L. de' Medici, G. Giovannetti, and M. Capone, Phys. Rev. Lett. 112, 177001 (2014).

[7] R. M. Fernandes, A. V. Chubukov, and J. Schmalian, Nat. Phys. 10, 97 (2014).

[8] S. Avci, O. Chmaissem, J. M. Allred, S. Rosenkranz, I. Eremin, A. V. Chubukov, D. E. Bugaris, D. Y. Chung, M. G. Kanatzidis, J.-P. Castellan et al., Nat. Commun. 5, 3845 (2014).

[9] A. E. Böhmer, F. Hardy, L. Wang, T. Wolf, P. Schweiss, and C. Meingast, Nat. Commun. 6, 7911 (2015).

[10] B. P. P. Mallett, C. N. Wang, P. Marsik, E. Sheveleva, M. Yazdi-Rizi, J. L. Tallon, P. Adelmann, T. Wolf, and C. Bernhard, Phys. Rev. B 95, 054512 (2017).

[11] J. G. Storey, J. W. Loram, J. R. Cooper, Z. Bukowski, and J. Karpinski, Phys. Rev. B 88, 144502 (2013).

[12] B. P. P. Mallett, P. Marsik, M. Yazdi-Rizi, T. Wolf, A. E. Böhmer, F. Hardy, C. Meingast, D. Munzar, and C. Bernhard, Phys. Rev. Lett. 115, 027003 (2015).

[13] J. M. Allred, K. M. Taddei, D. E. Bugaris, M. J. Krogstad, S. H. Lapidus, D. Y. Chung, H. Claus, M. G. Kanatzidis, D. E. Brown, J. Kang et al., Nat. Phys. 12, 493 (2016).

[14] J. Kang, X. Wang, A. V. Chubukov, and R. M. Fernandes, Phys. Rev. B 91, 121104(R) (2015).

[15] X. Wang, J. Kang, and R. M. Fernandes, Phys. Rev. B 91, 024401 (2015).

[16] J. Lorenzana, G. Seibold, C. Ortix, and M. Grilli, Phys. Rev. Lett. 101, 186402 (2008).

[17] M. N. Gastiasoro and B. M. Andersen, Phys. Rev. B 92, 140506(R) (2015).

[18] B. P. P. Mallett, Y. G. Pashkevich, A. Gusev, T. Wolf, and C. Bernhard, Europhys. Lett. 111, 57001 (2015).
[19] M. M. Qazilbash, J. J. Hamlin, R. E. Baumbach, L. Zhang, D. J. Singh, M. B. Maple, and D. N. Basov, Nat. Phys. 5, 647 (2009).

[20] D. H. Lu, M. Yi, S.-K. Mo, A. S. Erickson, J. Analytis, J.-H. Chu, D. J. Singh, Z. Hussain, T. H. Geballe, I. R. Fisher et al., Nature (London) 455, 81 (2008).

[21] A. Charnukha, S. Thirupathaiah, V. B. Zabolotnyy, B. Büchner, N. D. Zhigadlo, B. Batlogg, A. N. Yaresko, and S. V. Borisenko, Sci. Rep. 5, 10392 (2015).

[22] L. Ortenzi, E. Cappelluti, L. Benfatto, and L. Pietronero, Phys. Rev. Lett. 103, 046404 (2009).

[23] L. Benfatto and E. Cappelluti, Phys. Rev. B 83, 104516 (2011).

[24] L. Fanfarillo, J. Mansart, P. Toulemonde, H. Cercellier, P. Le Fèvre, F. Bertran, B. Valenzuela, L. Benfatto, and V. Brouet, Phys. Rev. B 94, 155138 (2016).

[25] D. V. Evtushinsky, V. B. Zabolotnyy, T. K. Kim, A. A. Kordyuk, A. N. Yaresko, J. Maletz, S. Aswartham, S. Wurmehl, A. V. Boris, D. L. Sun et al., Phys. Rev. B 89, 064514 (2014).

[26] L. Boeri, O. V. Dolgov, and A. A. Golubov, Phys. Rev. Lett. 101, 026403 (2008).

[27] L. Boeri, M. Calandra, I. I. Mazin, O. V. Dolgov, and F. Mauri, Phys. Rev. B 82, 020506(R) (2010).

[28] T. Egami, B. V. Fine, D. Parshall, A. Subedi, and D. J. Singh, Adv. Condens. Matter Phys. 2010, 164916 (2010).

[29] S. Coh, M. L. Cohen, and S. G. Louie, Phys. Rev. B 94, 104505 (2016).

[30] A. Akrap, J. J. Tu, L. J. Li, G. H. Cao, Z. A. Xu, and C. C. Homes, Phys. Rev. B 80, 180502(R) (2009).

[31] A. A. Schafgans, B. C. Pursley, A. D. LaForge, A. S. Sefat, D. Mandrus, and D. N. Basov, Phys. Rev. B 84, 052501 (2011).

[32] B. Xu, H. Xiao, B. Gao, Y. H. Ma, G. Mu, P. Marsik, E. Sheveleva, F. Lyzwa, Y. M. Dai, R. P. S. M. Lobo et al., Phys. Rev. B 97, 195110 (2018).

[33] B. Xu, Y. M. Dai, B. Shen, H. Xiao, Z. R. Ye, A. Forget, D. Colson, D. L. Feng, H. H. Wen, C. C. Homes et al., Phys. Rev. B 91, 104510 (2015).

[34] R. Yang, Y. Dai, B. Xu, W. Zhang, Z. Qiu, Q. Sui, C. C. Homes, and X. Qiu, Phys. Rev. B 95, 064506 (2017).

[35] A. E. Karkin, T. Wolf, and B. N. Goshchitskii, J. Phys. Condens. Matter 26, 275702 (2014).

[36] C. C. Homes, M. Reedyk, D. A. Cradles, and T. Timusk, Appl. Opt. 32, 2976 (1993).

[37] M. Dressel and G. Grüner, Electrodynamics of Solids (Cambridge University Press, Cambridge, England, 2002).

[38] L. Benfatto, E. Cappelluti, L. Ortenzi, and L. Boeri, Phys. Rev. B 83, 224514 (2011).

[39] A. Charnukha, J. Phys. Condens. Matter 26, 253203 (2014).

[40] P. Marsik, C. N. Wang, M. Rössle, M. Yazdi-Rizi, R. Schuster, K. W. Kim, A. Dubroka, D. Munzar, T. Wolf, X. H. Chen et al., Phys. Rev. B 88, 180508(R) (2013).

[41] M. J. Calderón, L. de Medici, B. Valenzuela, and E. Bascones, Phys. Rev. B 90, 115128 (2014).

[42] See Supplemental Material at http://link.aps.org/ supplemental/10.1103/PhysRevLett.122.217002 for details of the temperature-dependent optical conductivity spectra and the Fano fits, as well as the theoretical calculations of 
electron-phonon coupling of the $E_{u}$ mode in layered ironbased pnictides.

[43] W. Z. Hu, J. Dong, G. Li, Z. Li, P. Zheng, G. F. Chen, J. L. Luo, and N. L. Wang, Phys. Rev. Lett. 101, 257005 (2008).

[44] D. Wu, N. Barišić, P. Kallina, A. Faridian, B. Gorshunov, N. Drichko, L. J. Li, X. Lin, G. H. Cao, Z. A. Xu et al., Phys. Rev. B 81, 100512(R) (2010).

[45] A. Charnukha, D. Pröpper, T. I. Larkin, D. L. Sun, Z. W. Li, C. T. Lin, T. Wolf, B. Keimer, and A. V. Boris, Phys. Rev. B 88, 184511 (2013).

[46] Y. M. Dai, B. Xu, B. Shen, H. Xiao, H. H. Wen, X. G. Qiu, C. C. Homes, and R. P. S. M. Lobo, Phys. Rev. Lett. 111, 117001 (2013).

[47] B. Xu, Y. M. Dai, H. Xiao, B. Shen, H. H. Wen, X. G. Qiu, and R. P. S. M. Lobo, Phys. Rev. B 96, 115125 (2017).

[48] U. Fano, Phys. Rev. 124, 1866 (1961).

[49] E. Cappelluti, L. Benfatto, and A. B. Kuzmenko, Phys. Rev. B 82, 041402(R) (2010).

[50] E. Cappelluti, L. Benfatto, M. Manzardo, and A. B. Kuzmenko, Phys. Rev. B 86, 115439 (2012).

[51] T. Morinari, E. Kaneshita, and T. Tohyama, Phys. Rev. Lett. 105, 037203 (2010).

[52] J. M. Tranquada, B. J. Sternlieb, J. D. Axe, Y. Nakamura, and S. Uchida, Nature (London) 375, 561 (1995).

[53] E. Fradkin, S. A. Kivelson, and J. M. Tranquada, Rev. Mod. Phys. 87, 457 (2015).
[54] M. J. Rice and H.-Y. Choi, Phys. Rev. B 45, 10173 (1992).

[55] A. B. Kuzmenko, L. Benfatto, E. Cappelluti, I. Crassee, D. van der Marel, P. Blake, K. S. Novoselov, and A. K. Geim, Phys. Rev. Lett. 103, 116804 (2009).

[56] M. Daghofer, A. Moreo, J. A. Riera, E. Arrigoni, D. J. Scalapino, and E. Dagotto, Phys. Rev. Lett. 101, 237004 (2008).

[57] M. J. Calderón, B. Valenzuela, and E. Bascones, Phys. Rev. B 80, 094531 (2009).

[58] L. Fanfarillo, A. Cortijo, and B. Valenzuela, Phys. Rev. B 91, 214515 (2015).

[59] A. Kreisel, B. M. Andersen, P. O. Sprau, A. Kostin, J. C. S. Davis, and P. J. Hirschfeld, Phys. Rev. B 95, 174504 (2017).

[60] L. Classen, R.-Q. Xing, M. Khodas, and A. V. Chubukov, Phys. Rev. Lett. 118, 037001 (2017).

[61] R. Nourafkan, G. Kotliar, and A.-M. S. Tremblay, Phys. Rev. Lett. 117, 137001 (2016).

[62] L. Fanfarillo, L. Benfatto, and B. Valenzuela, Phys. Rev. B 97, 121109(R) (2018).

[63] L. Benfatto, B. Valenzuela, and L. Fanfarillo, npj Quantum Mater. 3, 56 (2018).

[64] S. Maiti, T. A. Maier, T. Böhm, R. Hackl, and P. J. Hirschfeld, Phys. Rev. Lett. 117, 257001 (2016).

[65] T. Böhm, F. Kretzschmar, A. Baum, M. Rehm, D. Jost, R. Hosseinian Ahangharnejhad, R. Thomale, C. Platt, T. A. Maier, W. Hanke et al., npj Quantum Mater. 3, 48 (2018). 